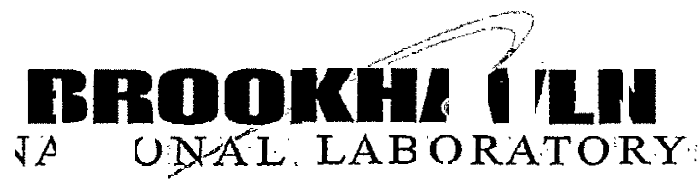

BNL-73382-2005-CP

\section{IBSfor Ion Distribution under Electron Cooling}

\author{
A.V. Fedotov, I. Ben-Zvi, Yu. Eidelman, \\ V.N. Litvinenko, G. Parzen, et. al.
}

\author{
To be presented at Particle Accelerator Conference \\ Knoxville, Tennessee \\ May 16-20, 2005
}

Collider-Accelerator Department

\section{Brookhaven National Laboratory}

P.O. Box 5000

Upton, NY I1973-5000

www:bnl:gov

Managedby .

Brookhaven Science Associates, LLC

for the United States Department of Energy under

Contract No. DE=AC02-98CH10886 


\section{DISCLAIMER}

This report was prepared as an account of work sponsored by an agency of the United . States Government. Neither the United States Government nor. any agency thereof, nor any of their employees, nor any of their. contractors; subcontractors, or their employees, makes any warranty, express or implied, or assumes, any legal liability or responsibility for the accuracy, completeness, or any third party's use or the results of such use of any information, apparatus, product, or process disclosed, or represents that its use would'not infringe privately owned rights. Reference herein.to any specific commercial.product, process, or service by trade name, trademark, manufacturer, or: otherwise, does not necessarily constitute or imply its endorsement, recommendation; .or favoring by the United States Government or any agency thereof or its contractors or subcontractors. The views and opinions of authors expressed herein do not necessarily state or reflect those of the United States Government or any agency thereof 


\title{
IBS FOR ION DISTRIBUTION UNDER ELECTRON COOLING*
}

\author{
A.V.Fedotov", I. Ben-Zvi, Yu. Eidelman, V. N. Litvinenko, G. Parzen, BNL, Upton, NY 11973 \\ A. Sidorin, A. Smirnov, G. Trubnikov, JINR, Dubna, Russia
}

\section{Abstract}

Standard models of the intra-beam scattering (IBS) are based on the growth of the rms beam parameters for a Gaussian distribution. As a result of electron cooling; the core of beam distribution is cooled much faster than the tails, producing a denser core. In this paper, we compare various approaches to IBS treatment for such distribution. Its impact on the luminosity is also discussed.

\section{CORE-TAIL MODEL}

Formation of a dense core due to cooling and diffusion is modeled using the macro-particle :approach which allows variable with time beam distribution. The individual-particle kicks due to cooling and IBS are applied in the velocity space. Such approach was the; basis of the SIMCOOL code [1] and was also recently implemented in the BETACOOL code as a "Model Beam" approach [2] : To account for a core collapse (which directly impacts luminosity in a collider) of ion distribution, the "core-tail". model for the IBS was proposed. In general, to describe dynamics of such distributions an accurate kinetic simulation is required which vill be addressed in the future work. With the core- $:$ tail model we attempt to capture only basic features of the core formation in order to estimate the luminosity. In this. model, the individual-particle kick in the velocity space due to IBS is applied based on diffusion coefficients which are different for particles inside and outside of the core. Cooling process for typical RHIC parameters [3] is shown in Figs. 1-4. Figure 1 shows that an $\mathrm{rms}$ emittance under cooling. stays approximately constant for these parameters, while there is a fast formation of a distinct core in the beam profiles.

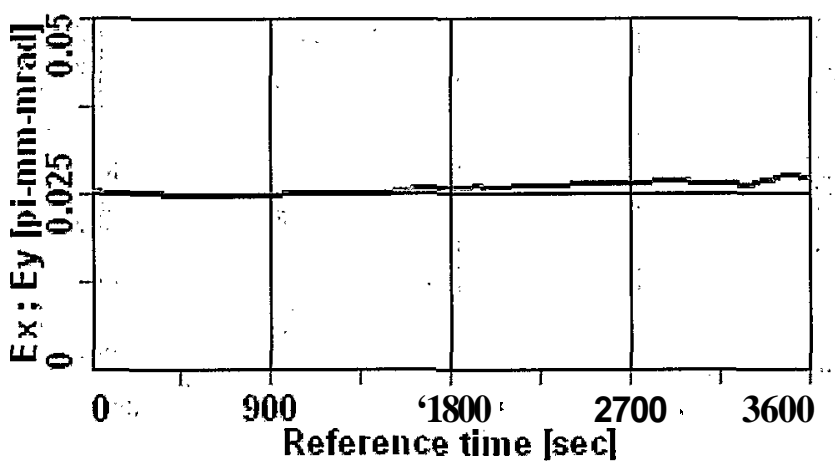

Figure 1: Time evolution of unnormalized transverse rms emittance for typical magnetized cooling parameters of $\mathrm{Au}$ ions at $100 \mathrm{GeV} / \mathrm{u}$.

*Work supportedby the U.S. Department of Energy \#fedotov@bnl.gov.

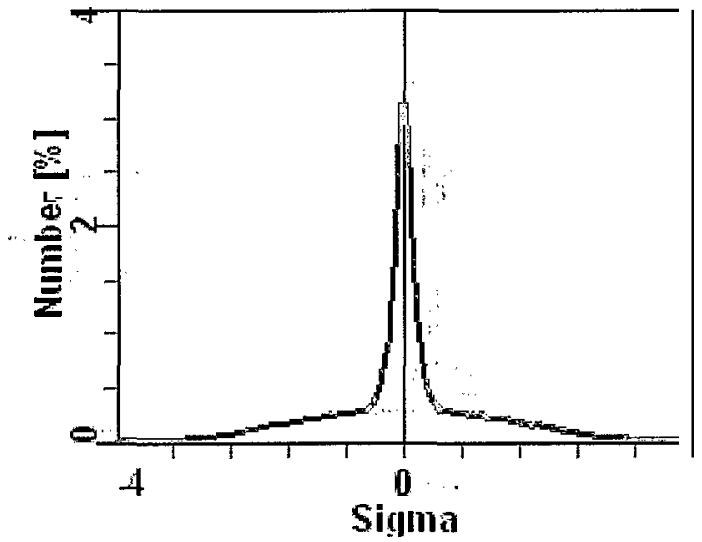

Figure 2: Transverse profile of ion beam after 1 hour of cooling with the IBS calculation based on tms values of full distribution.

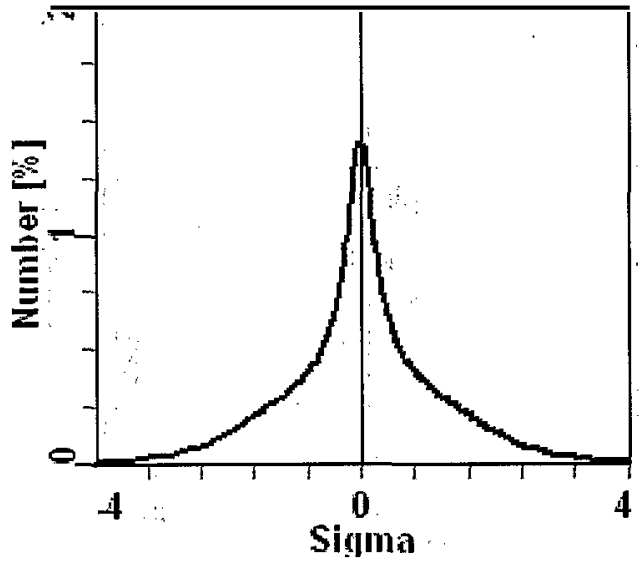

Figure 3: Transverse profile of ion beam after 1 hour of cooling with the "core-tail" IBS approach.

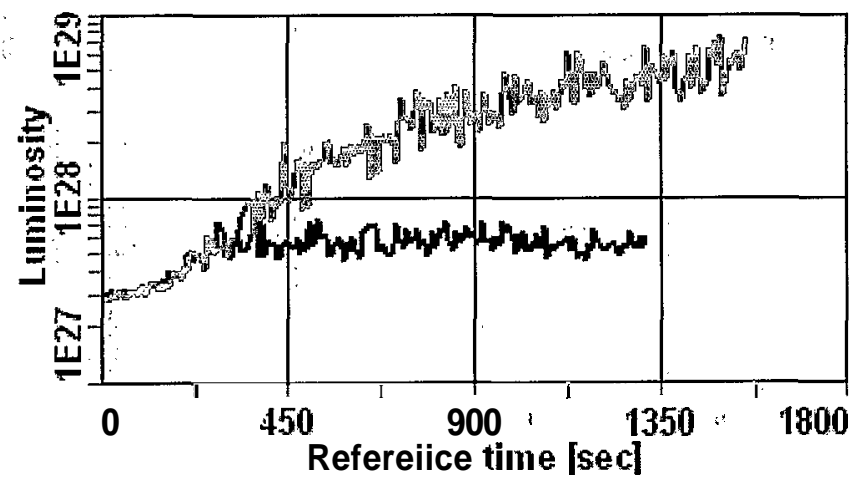

Figure 4: Luminosity growth using IBS approach based the rms of full distribution (red top curve) and using "core-tail" approach (blue), for typical parameters of the magnetized cooling for $\mathrm{Au}$ ions at $100 \mathrm{GeV} / \mathrm{u}$. 
For the distribution with a pronounced core, if one calculates the rms parameters of a full distribution (with $; \ldots$ tails) and uses them to calculate the IBS kicks on all particles, the IBS for particles in the core may be strongly underestimated. Figure 2 shows formation of a "collapsed" core when the IBS kicks are applied based on the rms parameters calculated for a full. distribution (which are approximately constant for chosen parameters, see Fig. 1). This calls for a different treatment of particles in the core and tails. Figure 3 shows beam distribution when IBS kicks are applied according to the "core-tail". model. The predicted luminosity based on these two approaches is very different, as shown in Fig. 4 (with logarithmic scale on the "verticalaxis).

To demonstrate how the core-tail model works we use simplified expressions for the diffusion coefficients based on the gas-relaxation formula:[4,5]: In an approximation that the transverse temperature of the ion beam is much . higher than the longitudinal, the longitudinal coefficient can be easily derived [6]: Such approximation is valid for energies much higher than the transition energies as can be seen from the flatness parameter which is defined as a ratio of beam temperatures in the beam moving frame. As a result, this parameter describes a degree of flatness of the distributionfunction in the velocity space:

$$
g_{f}=\left(\frac{v_{\text {longind }}}{v_{\text {transverse }}}\right)^{2}=\frac{\sigma_{p}^{2}}{\gamma^{2}\left(\varepsilon / \beta_{a}\right)},
$$

where $\sigma_{\mathrm{p}}$ is an rms momentum spread, $\varepsilon$ is an rms emittance, and $\beta_{\mathrm{a}}$ is the beta function.averaged over the ring. For typical parameters of $\mathrm{Au}$ ions in RHIC at 100 $\mathrm{GeV} / \mathrm{u}$ the flatness parameter is $0.1-0.2$, and assumption that the distribution is flat $\left(v_{\text {transverse }} \gg v_{\text {longitudinal }}\right)$ may be used. In such an approximation, the diffusion : coefficient . in the longitudinal direction can be written for a bunched beam as [5]:

$$
D_{z z}=\frac{N r^{2} c}{8(\gamma \beta)^{3}} \frac{\Lambda_{i b s}}{\varepsilon_{x}^{3 / 2} \sqrt{\beta_{a}} \sigma_{s}} \sqrt{\frac{2}{\pi}}=N \frac{C}{\varepsilon_{x}^{3 / 2} \sigma_{s}}
$$

where $\mathrm{E}$ is the horizontal $\mathrm{rms}$ beam emittance, $\sigma_{\mathrm{s}}$ is an rms bunch length, $\beta_{\mathrm{a}}$ is an average beta-function over the ring lattice, $r$ is the classical radius, $\Lambda_{\text {ibs }}$ is the Coulomb logarithm for IBS, and $\mathrm{N}$ is the total number of particles in a bunch. Note, that one gets exactly the same coefficient apart from a factor $(2 / \pi)^{1 / 2}$, using the highenergy approximation of Bjorken-Mtingwa [7] formulas for the IBS with an assumption of a smooth lattice [5]: In the "core-tail" model one finds rms parameters separately for the core $\left(\varepsilon_{c}, \sigma_{s c}\right)$ and tails $\left(\boldsymbol{E}, \sigma_{s}\right)$ of the distribution. These rms parameters are then used to apply different diffusionkicks for particles which are in the core and tails according to the expressions

$$
D_{z z, \text { core }}=N_{\text {core }} \frac{C}{\varepsilon_{c}^{3 / 2} \sigma_{s c}}+\left(N-N_{\text {core }}\right) \frac{C}{\varepsilon^{3 / 2} \sigma_{s}}
$$

$$
D_{z z, \text { tails }}=N \frac{C}{\varepsilon^{3 / 2} \sigma_{s}} .
$$

The accuracy of the algorithm depends on finding a number of particle in the core of the distribution $\mathrm{N}_{\text {core }}$ and an rms parameters for the core and tails. In the first simplified approach, the core parameters were determined through the FWHM of the distribution while an rms parameters of the full distribution were used for the tails. This model was later improved with a numerical procedure which fits two Gaussian distributions to a real distribution observed in simulations for each time step of the calculation. The amplitude and width of fitted Gaussians (in all three directions) provide more accurate parameters which are used instead of E, $\varepsilon_{\mathrm{c}}, \sigma_{\mathrm{s}}, \sigma_{\mathrm{sc}}$ in the .diffusion : coefficients. In the, same approximation of the high energy, when heating is dominated by a longitudinal degree of freedom, the transverse diffusion rate can be expressed through the longitudinal one using the $\mathrm{H}$ function of the ring (for a smooth lattice it is $\left\langle\mathrm{D}_{\mathrm{x}}{ }^{2} / \beta_{\mathrm{x}}\right\rangle$ ):

$$
\tau_{\perp}^{-1}=\frac{\sigma_{p}^{2}}{\varepsilon_{x}}\left\langle\frac{D_{x}^{2}+\left(D_{x}^{\prime} \beta_{x}+\alpha_{x} D_{x}\right)^{2}}{\beta_{x}}\right\rangle \tau_{I I}^{-1},
$$

where $\sigma_{p}$ is the rms momentum spread, $D_{x}, D^{\prime}, \alpha_{x}, \beta_{x}$ are the lattice functions, $<>$ stands for averaging over the ring, and the longitudinal growth rate is defined as

$$
\tau_{I I}^{-1}=\frac{1}{\sigma_{n}^{2}} D_{z z} \text {. }
$$

More general relations, which are valid for all energies and. include derivatives of the lattice functions are summarized in $[7,8,9]$. In fact, for accurate simulation of the IBS in RHIC we do use one of the expressions from $[7,8,9]$, all of which are implemented in the BETACOOL code, and were recently cross-checked vs. one another [10]: Experimental verification. of these models vs. dedicated experiments of IBS at RHIC [11] with good agreement, increased our confidence in numeric models being used. The "core-tail" approach is also implemented in the BETACOOL code. As a result, it allows us to use more accurate diffusion coefficients (rather than the one in Eq. (2)) which can take into account correct ratio * between the temperatures of the ion beam, as well as realistic RHIC lattice (including derivatives). In addition, thestandard IBS theory was recently reformulated for the growth rates of a bi-Gaussian distribution [12].

\section{DIFFUSION COEFFICIENTS}

The "core-tail": model described in previous section is just an application of standard IBS theory $[7,8,9]$ for the distribution which has a pronounced core with an attempt to have an estimate for expected luminosity. Also, it does not take into account dependence of the diffusion coefficients on particle amplitudes within the core. Below we try to explore the accuracy of such assumption..

A detailed treatment of the IBS, which depends on individual particle-amplitudes, was recently proposed by Burov [13], with an analytic formulation done for a 
Gaussian distribution in approximation that transverse rms velocity of the ion beam is much higher than the longitudinal .In : general, for accurate dependence of the diffusion $\cdots$ coefficients $:$ on both the transverse and longitudinal velocities, which are needed to describe IBS for different beam parameters at different energies (which is one of the tasks for RHIC" since cooling at various energies is considered [14]); the integrals over the distribution function should be performed numerically. Similar .. algorithm was recently implemented : for numerical calculation of the non-magnetized friction force. [2], for example. For accurate-IBS calculation one has to: calculate such integrals at each lattice element which. would: make calculations too. slow: However, similar approach is presently: being considered for a simplified lattice structure in order to perform needed benchmarking of the core-tail model:

In the "core-tail" model, the diffusion coefficients are found at each lattice element for the particles in the core and tails of beam distribution: However, the assumption is made that all particle in the core get the same kick compared to the amplitude-dependent coefficient within the core. To understand the accuracy of this approximation one can have a look at the dependence of the diffusion coefficient on amplitude. For anisotropic Maxwellian distribution written in the form: ...

$$
f(v)=\frac{n}{\pi \sqrt{2 \pi} \cdot \Delta^{2} \mathbf{A}_{I I}} \mathrm{e}^{-v^{2}{ }_{\perp} / \Delta^{2} \perp-v_{I I}^{2} /\left(2 \Delta^{2} I I\right)}
$$

the longitudinal component of the diffusion tensor is given by .

$$
D_{z z}=\frac{4 \pi n(Z e)^{4}}{m^{2}} \Lambda_{i h \cdot} \int d^{3} v f(\vec{v}) \frac{w^{3}-w t z}{w^{2}},
$$

with $\vec{u}=u \hat{x}$ and $\vec{w}=\mathrm{v}-u \hat{x}$. When one assumes that an rms: transverse velocity is much larger than the longitudinal one.(flattened distribution); the integrals can be easily evaluated,.as shown, for example, by Sorensen [5], with the result:

$$
D_{z z}=\frac{4 \pi n(Z e)^{4}}{m^{2} \Delta_{\perp}} \Lambda_{i b s}\left[\sqrt{\pi} e^{-u^{2} / 2 \Delta^{2} \perp} I_{0}\left(\frac{u^{2}}{2 \Delta_{\perp}^{2}}\right)\right]
$$

A generalization of this expression to include also dependence on spatial amplitudes was done by Burov [13]. The function in square brackets in Eq. (9) decreases very slowly with the amplitude $u$. For $u=0$, it gives a factor $\sqrt{\pi}$, while for amplitudes equal to an rms value $\Delta_{\perp}$, the expression in the square brackets is close to unity, which allows us to approximate the amplitude dependence by an rms values with a reasonably good accuracy. The expression in Eq. (9) is then simply becomes:

$$
D_{z z}=\frac{4 \pi n(Z e)^{4}}{m^{2} \Delta_{\perp}} \Lambda_{i b s}
$$

When rewritten in terms of beam parameters for the bunched beam, Eq: (10) gives Eq. (2). As a result, it seems that an assumption of a constant-amplitude kick based on the rms values should give reasonably accurate estimate.: At the same time it allows us to do efficient computer calculations and include (in an accurate way) the ratio between the transverse and longitudinal beam velocities. without approximations. Also; such an assumption allows us fast calculation of the IBS : at each lattice element with a realistic lattice rather than:assuming a smooth approximation. The:formulas for "detailed" IBS by Burov. [13] were also included in the BETACOOL. Cooling dynamics results based on the "detailed" and "core-tail" approach were found qualitatively similar. However; it is not clear whether observed differences are related to the amplitude dependence or to the approximations being made in [13]. At this point, we are evaluating various : scenarios, including the " use of formalism in [12] for the diffusion coefficients, as well as a possibility of an accurate numerical calculation, to understand what is the most efficient and at the same time sufficiently accurate approach. Benchmarking: vs. 3D "IBS map" approach [15], as well as vs. experiments is also being considered.

\section{ACKNOWLEDGMENTS}

We would like to thank A. Burov, V. Lebedev, J. Wei and the Accelerator Physics Group of Electron Cooling Project of RHIC for many useful discussions during these studies. We also acknowledge collaboration on this subject with the INTAS project "Advanced Beam 'Dynamics for Storage Rings".

\section{REFERENCES}

[1] The SIMCOOL code was originally developed at BINP, Novosibirsk; see for details A.V. Fedotov et al., TPAT090 (these proceedings).

[2] The BETACOOL program; http://lepta.iinr.ru

[3] RHIC E-cooler Design Report ।

http://www. agsshichome.bnl.gov/eCool

[4] I. Ben-Zvi, V.V. Parkhomchuk, C-AD/AP/47 (2001).. *

[5] A.V. Fedotov, Tech. Note C-AD/AP/168 (2004).

[6]A. Sorensen, CERN Acc. School (1987).

[7] Bjorken and Mtingwa, Part. Acc., 13,p.115 (1983).

[8] M. Martini, CERN PS/84-9 (1984).

[9] A. Piwinski, CERNAS, CERN 85-19, p.451 (1985).

[10] G. Trubnikov, A.V. Fedotov (2004); unpublished.

[11] J. Wei et al., TPAT081, these proceedings (2004).

[12] G. Parzen, Tech. Note C-AD/AP/150 (2004).

[13] A. Burov, FERMILAB-TM-2213(2003): ‘

[14] A. V. Fedotov, TPAT089; these proceedings.

[15] P. Zenkevich et al., Proceedings of ICFA-HB2004 Workshop, Bensheim, Germany (2004). 